\title{
Atención de enfermería en niños con síndrome de Guillain-Barré, etapa aguda
}

\author{
Srtas. Gloria Carvajal Placencio y Rosa E. Ljubetic Rodríguez*
}

En el período que abarca desde el año 1972 hasta marzo del año en curso, se han presentado en la Unidad de Infecciosos del Servicio de Pediatría del Hospital Regional de Antofagasta, 22 casos clínicos del Síndrome de GuillainBarré, de los cuales cinco fallecieron, dos de ellos correspondían a la modalidad Landry.

Dada la escasa información bibliográfica existente en nuestro medio sobre el Síndrome de Guillain-Barré y de acuerdo con la casuística, anteriormente descrita, el profesional de enfermería se encuentra ante un desafio para proporcionar una atención adecuada a los múltiples problemas que afectan a este tipo de paciente y al riesgo latente de complicaciones que en su punto más grave conducen a la muerte. Este hecho nos motiva a confeccionar un plan de atención de enfermería para estos niños.

Considerando que este síndrome es una poliradiculoneuritis aguda con disociación albúmino-citológica del líquido cefalorraquídeo, que se manifiesta como un cuadro infeccioso agudo de evolución progresiva, con alteraciones motoras y sensitivas, mialgias, cefalea, alteraciones vasomotoras, alteraciones esfinterianas transitorias, irritabilidad y decaimiento; eva-

*Docentes de Enfermería, Depto. Ciencias de la Salud, U. de Chile - Sede Antofagasta. luaremos a este paciente como un individuo que presenta un estado físico-patológico recuperable con leve alteración del psiquis por temor a los problemas que le afectan.

\section{OBjetivos}

1. Identificar los problemas de salud que presentan los niños con Síndrome de GuillainBarré, hospitalizados en la Unidad de Infecciosos.

2. Elaborar un plan de cuidados de enfermería que sirva de pauta para la atención de estos pacientes por el profesional enfermera $(0)$.

\section{METODOLOGIA}

Revisión de. fichas clínicas de 22 casos de Síndromes de Guillain-Barré, presentados entre los años 1972 a marzo de 1979, en la Unidad de Infecciosos del Servicio de Pediatría del Hospital Regional Leonardo Guzmán de Antofagasta.

Las edades de estos niños fluctuaron entre un año seis meses y catorce años cinco meses, el $50 \%$ de ellos procedían de localidades como Siballa (precordillera Iquique), Calama, Tocopilla, Maria Elena, Mejillones.

El diagnóstico médico del síndrome se realizó en base al cuadro clínico y examen de la- 
boratorio citoquímico del líquido cefalorraquídeo.

\section{Resultados}

De la revisión de fichas clínicas se obtuvieron los siguientes datos:

Los trastornos que con mayor frecuencia debió atender el profesional de enfermería fueron los trastornos motores $(51,3 \%)$, dolores $(19,1 \%)$ y trastornos vasomotores $(8,6 \%)$ (Gráfico 1$)$.

Analizada la distribución de los signos y síntomas por trastornos se observa que la hipotonía

Gráfico $N^{\circ} 1$

Trastornos presentados en 22 casos clínicos de síndrome de Guillain-Barre.

Unidad Infecciosos-Servicio Pediatría Hospital Regional-Antofagasta. 1972 - Marzo - 1979.

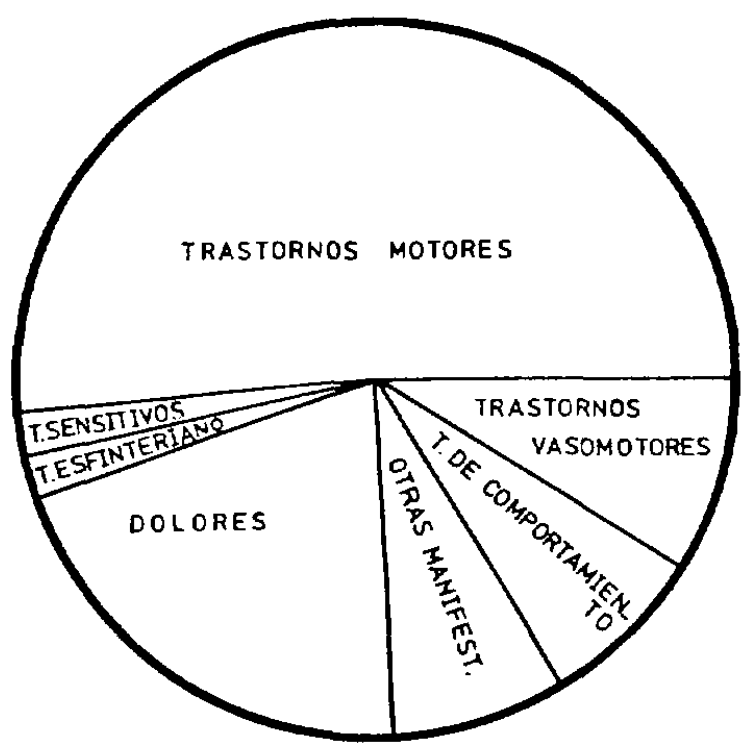

Trastornos motores

Dolores

$31.4 \%$

Trastornos sensitivos

$19.2 \%$

Trastornos esfinterianos

$2.6 \%$

Trastornos de comportamiento

Otras manifestaciones

Trastornos vasomotores

$2.6 \%$

$7.8 \%$

$7.8 \%$

$8.6 \%$ y la arreflexia tendinosa aparecen como los más frecuentes con 19 y 18 casos respectivamente, y la parálisis fláccida se presentó en 14 de los pacientes.

En relación a los dolores son las mialgias las que predominan considerablemente (17 pacientes) en cambio las raquialgias sólo están presente en cuatro de los casos estudiados.

Se denota un gran predominio de la taquicardia, nueve casos, sobre la bradicardia encontrada sólo en un paciente.

El decaimiento y la irritabilidad con cinco y cuatro casos se consignan como los trastornos del comportamiento observado en estos pacientes.

Debemos destacar que la mayoría de los pacientes tuvo la sensibilidad conservada y sólo tres de ellos presentaron hipoestesias (Gráfico 2).

Entre las complicaciones, el $22,7 \%$ fueron respiratorios (dos casos de Landry) y el $18,1 \%$ atrofia muscular. El $59 \%$ del universo evolucionó sin complicaciones (Gráfico 3).

Observamos que 17 de los pacientes $(77,3 \%)$ tuvieron regresión del cuadro clínico y cinco de ellos fallecieron $(22,7 \%)$, hecho que coincide con las complicaciones respiratorias presentadas (Gráfico 4).

La atención de enfermería que recibieron durante su hospitalización fue en 15 niños $(68,1 \%)$ inespecífica y sólo 7 pacientes $(31,8 \%)$ recibieron atención específica a sus necesidades. Sin embargo en un $45,4 \%$ del universo ( 10 casos) las observaciones de enfermería fueron referentes a sus problemas (Gráfico 5 ).

\section{CONGLUSiones}

Después de analizados los datos obtenidos de las fichas clínicas, se concluye que los cuidados de enfermería deben estar fundamentalmente orientados a la atención y control de las manifestaciones más frecuentes, sin descuidar la observación y atención de otros trastornos presentados por el paciente.

\section{PLAn de atención de enfermería}

1. En primer lugar la enfermera(o) se encuentra ante un paciente con síndrome paralítico cuya etiología no está aún determinada. 
Signos y sintomas presentados por los casos clínicos estudiados.

Unidad Infeccioso-Servicio Pediatría Hospital Regional-Antofagasta. 1972 - Marzo - 1979.

NUME $R O$

CASOS

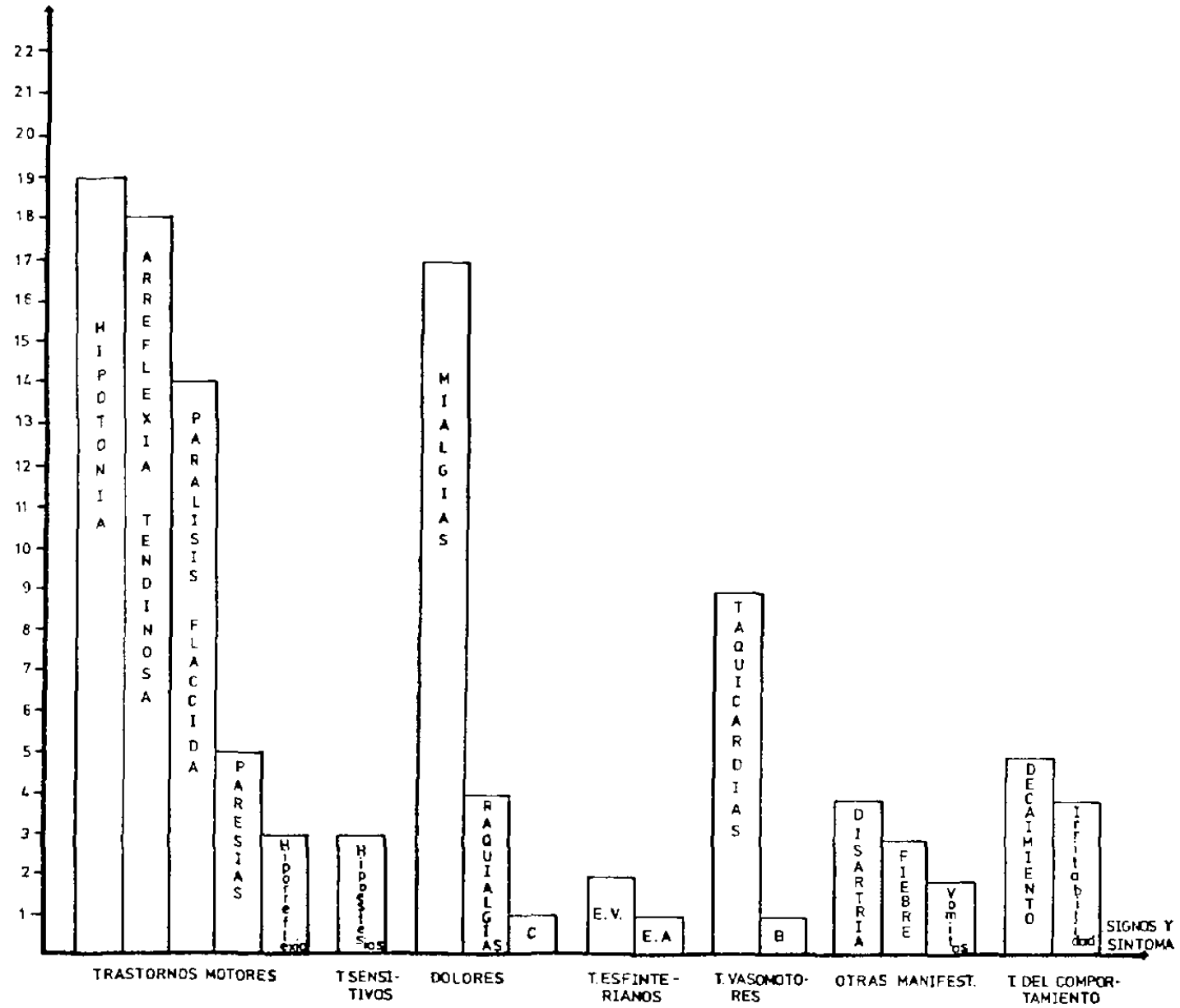

Gráfico $\mathrm{N}^{\circ} 3$

Complicaciones presentadas en 22 pacientes con sindrome de Guillain-Barre.

Unidad Infecciosos-Servicio Pediatría Hospital Regional-Antofagasta. 1972 - Marzo - 1979.

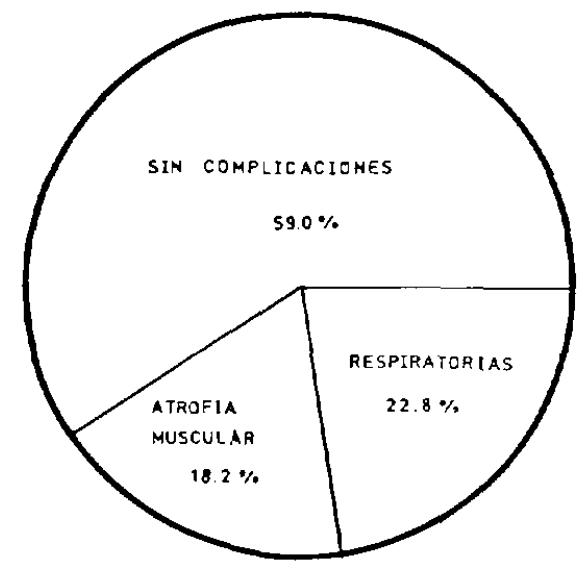

\subsection{Cuidados de enfermería}

1.1.1. Aislamiento del paciente hasta que se determine el diagnóstico diferencial con Poliomielitis.

1.1.2. Uso de técnicas de enfermería en aislamiento para pacientes con enfermedad trasmisible por vía digestiva.

1.1.3. Toma de muestras para exámenes de laboratorio:

a) sangre: para título virológico, 8 a $10 \mathrm{cc}$ en frasco estéril sin anticoagulante. La primera muestra se tomará en la etapa aguda y la segunda muestra en la etapa de convalecencia o 15 días después de la primera; 
Evolución de 22 casos clínicos con sindrome de Guillain-Barre. Unidad Infecciosos-Servicio Pediztría Hospital Regional-Antofagasta. 1972 - Marzo - 1979.

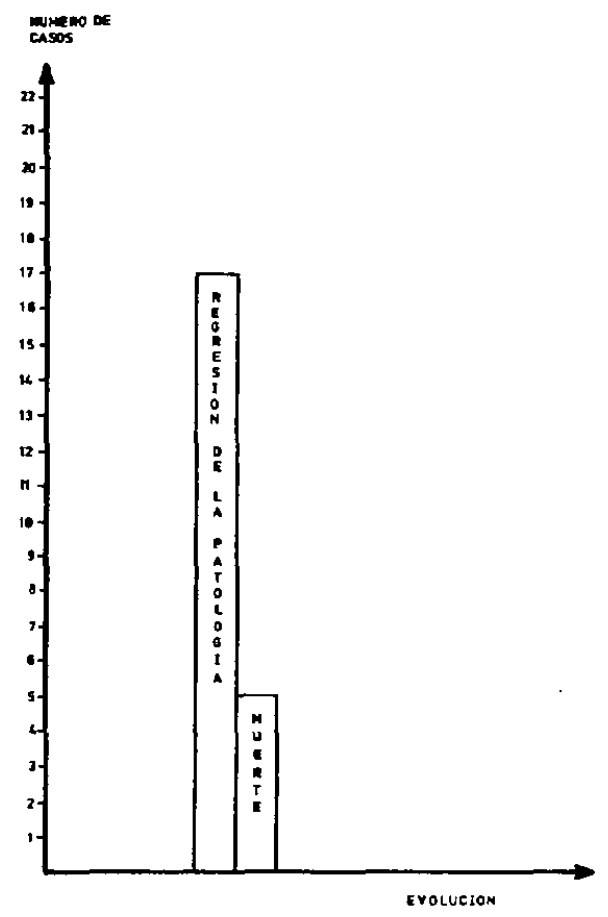

b) deposiciones: muestra seriada, día por medio, 3 muestras de 6 a 8 gramos cada una, y

c) colaboración en toma de muestra de LCR: 4 cc en frasco estéril para aislamiento del virus. Todas les muestras deben enviarse a laboratorio rodeadas de hielo.

NoTA: De acuerdo a las recomendaciones dadas por el Instituto Bacteriológico de Chile, en todo cuadro paralítico de etiología no confirmada deben adoptarse medidas de aislamiento del paciente y tomar muestras para examen de laboratorio necesarias para la determinación del agente etiológico.

2. Trastomos motores: se caracterizan por aparecer en forma simétrica desde los miembros inferiores. Estos son la hipotonia, arreflexia tendinosa, parálisis fláccida, paresias e hiporreflexias.

\subsection{Cuidados de enfermería}

2.1.1. Observar y evaluar la función motora de los miembros afectados. La evaluación se hace en tres rangos que son: con ayuda total, con ayu-
Grafico Nos

Tipo de arención de enfermería efectuada a los casos clínicos estudiados.

Unidad Infecciosos-Servicio Prematuros-Hospital Regional Antofagast2. 1972 - Marzo - 1979.

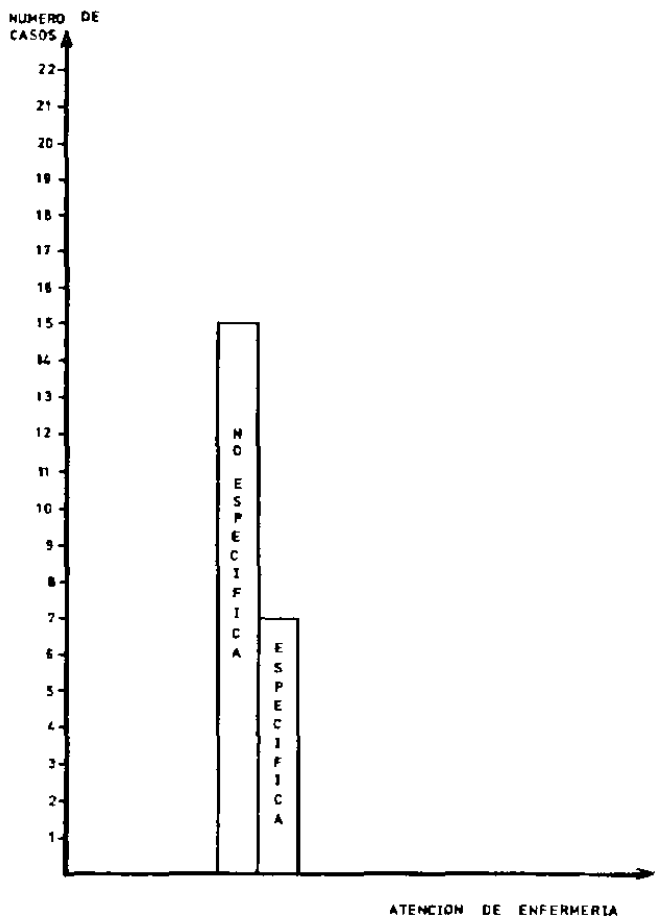

da parcial e independiente, basándose en la capacidad funcional del cuello, extremidades inferiores y superiores.

La capacidad funcional del cuello se evalúa mediante la flexión.

Para evaluar la funcionalidad de las extremidades superiores tenemos los movimientos de:

a) elevación de la mano hasta la cabeza y por detrás de ella;

b) aducción del brazo lateralmente hasta el nivel del hombro;

c) pronación y supinación del antebrazo;

d) capacidad de prehensión y su iniciación, y

e) cerrar y abrir el puño.

La actividad motora de las extremidades inferiores se mide a través de:

a) flexión y extensión de la rodilla;

b) tocar el suelo en posición sentada;

c) cruzar la pierna sobre la rodilla opuesta en posición sentada (con o sin ayuda de las manos);

d) paso de posición sentada a erecta (con o sin ayuda), y 
e) ambulación.

Registrar las observaciones en la hoja de valoración del funcionamiento del paciente (ver hoja en página siguiente).

2.1.2. Cambios de posición cada quince minutos.

2.1.3. Masajes para activar la circulación de los músculos afectados.

2.1.4. Prevención de úlcer as de decúbito.

2.1.5. Mantener la posición fisiológica de los miembros afectados, con bolsas de arena, férul as, o almohadillas (ver ilustración en pág. ).

2.1.6. Movilización pasiva de los miembros comprometidos, ejecutando ejercicios de flexión, extensión, abducción, aducción, supinación, pronación, rotación interna y externa (ver ilustración en pág. ).

3. Dolores: las manifestaciones dolorosas más frecuentes son las musculares (mialgias) y en menor frecuencia raquialgias.

\subsection{Cuidados de enfermería}

3.1.1. Cama dura y alza ropa.

3.1.2. Aplicación de compresas de agua tibia cada 6 u 8 horas.

3.1.3. Baño tibio diario.

Las medidas anteriores tienen por objeto contribuir a la relajación muscular y al aumento de la circulación de la masa muscular afectada y de esta manera disminuir el dolor.

4. Fenómenos vasomotores: de estos trastornos la enfermera(o) debe pesquisar las taquicardias y bradicardias. Podrían aparecer también hipertensión arterial y cambios de coloracion de la piel como el rubor.

\subsection{Cuidados de enfermería}

4.1.1. Control de pulso cada 4 horas o según necesidad (frecuencia, ritmo, amplitud).

4.1.2. Control de presión arterial cada 4 horas o según necesidad.

4.1.3. Observar y registrar cambios de coloración de la piel.

5. Complicaciones respiratorias: estas pueden ir desde el acúmulo de secreciones hasta el paro respiratorio por parálisis de pared costal. La enfermera(o) deben estar preparada para pesquisar y actuar en forma oportuna ante cualquier emergencia respiratoria.

\subsection{Cuidados de enfermería}

5.1.1. Control de la dinámica respiratoria cada dos horas.

VALORACION DEL FUNGIONAMIENTO DEL PACIENTE

\begin{tabular}{|c|c|c|c|}
\hline Capacidades funcionales & $\begin{array}{c}\text { Ayuda } \\
\text { total }\end{array}$ & $\begin{array}{l}\text { Ayuda } \\
\text { parcial }\end{array}$ & $\begin{array}{c}\text { Indepen- } \\
\text { diente }\end{array}$ \\
\hline $\begin{array}{l}\text { 1. Flexión del cuello } \\
\text { 2. Elevar la mano hasta la cabeza } \\
\text { 3. Elevar la mano por detrás de la cabeza } \\
\text { 4. Aducción del brazo que llega hasta el nivel del hombro } \\
\text { (lateralmente) } \\
\text { 5. Pronación supinación del antebrazo } \\
\text { 6. Prehensión de objetos } \\
\text { 7. Iniciación de capacidad de prehensión } \\
\text { 8. Cerrar el puño } \\
\text { 9. Abrir el puño } \\
\text { 10 Flexión y extensión de rodilla } \\
\text { 11. Tocar el suelo en posición sentada } \\
\text { 12. Cruzar la pierna sobre la rodilla opuesta en posición } \\
\text { sentada (con o sin ayuda de las manos) } \\
\text { 13. Paso de posición sentada a erecta (con ayuda de sostén } \\
\text { o sin ella) } \\
\text { 14. Ambulación }\end{array}$ & & & \\
\hline
\end{tabular}


a) frecuencia

b) ritmo

c) movimientos respiratorios

d) coloración de piel y mucosas.

5.1.2. Mantener vía aérea permeable.

a) aspiración de secreciones

b) ejercicios respiratorios.

5.1.3. Posición de semifowler.

5.1.4. Disponer de equipos de:

a) intubación

b) traqueotomía

c) oxigenoterapia

d) pullmotor.

6. Trastomos del comportamiento: las manifestaciones observadas en los niños son el decaimiento y la irritabilidad, derivados de los problemas físicos y psicosociales a los que se enfrenta.

\subsection{Cuidados de enfermería}

6.1.1. Habitación tranquil a y ventilada.

6.1.2. Brindar apoyo emocional al niño $y$ grupo familiar.

6.1.3. Proporcionar recreación y estímulos agradables. Aunque el paciente esté totalmente inmóvil se le debe consultar sobre sus preferencias y alentarlo a recibir alguna recreación como música, conversación o lectura.

6.1.4. Informar al paciente $y / 0$ familiares sobre la patología y atención de enfermería que se le proporciona.

7. Trastomos sensitios: aunque en el universo estudiado sólo hubo tres casos que presentaron hipoestesias, la enfermera(o) debe tener presente que pueden aparecer también parestesia distales o peribucales y disestesias.

\subsection{Cuidados de enfermería}

7.1.1. Observar y evaluar la sensibilidad de los miembros comprometidos, que tiene por objeto medir las sensaciones cutáneas superficiales y profundas, al igual que la capacidad de reconocer objetos por el tacto.

Los métodos de evaluación que pueden utilizarse son:

a) Estimulación con frío y calor: aproximar a la región a evaluar un tubo de ensayo con agua fría o caliente ( $\sin$ que el paciente conozca la temperatura del agua) y se pregunta la sensación que percibe. b) Estimulación con hisopillo de algodón: se roza suavemente la piel de la región a evaluar (paciente con vista vendada) y se interroga si percibe algún estímulo.

c) Estimulación con afiler: se realiza con el paciente con vista vendada y se punciona superficialmente el sitio a evaluar, interrogando sobre la percepción.

d) Estimulación con horquillas: cuyas distancias entre sus extremos deben ser de medio centímetro, uno y dos centímetros. Se realiza la prueba estando el paciente con la vista vendada y se pregunta cuántos puntos siente. Cuando el paciente percibe ambas puntas del estimulador menor, mejor es su percepción.

8. Trastomos esfinterianos transitorios: ocasionalmente pueden presentarse alteraciones de los esfinteres vesical $y / o$ anal, que puede ser la relajación del esfinter vesical, o constipación.

Al observar el control esfinteriano en el nino debemos tener presente la edad y los antecedentes del control voluntario del esfínter tanto diurno como nocturno. Del esfínter anal la enfermera(o) debe conocer las características de la defecación normal del paciente, fundamentalmente en relación a control voluntario $\mathrm{y}$ frecuencia de eliminación.

\subsection{Cuidados de enfermería}

8.1.1. Observar el control esfinteriano y pesquisar anomalías en él.

8.1.2. Instalación de recolector de orina en caso necesario.

8.1.3. Aseo genital según necesidad.

8.1.4. Lubricación región genital.

8.1.5. Cambio de muda y ropa de cama.

8.1.6. Estimulación de eliminación

de heces a través de ingestión adecuada de líquidos, dieta con residuos, ejercicios activos o pasivos dentro de los límites impuestos por la enfermedad.

9. Pesquisa y observación de otras manifestaciones como fiebre, vómitos, etc.

\subsection{Cuidados de enfermería}

9.1.1. Control de temperatura corporal cada cuatro horas.

9.1.2. Medidas físicas sobre $38,5^{\circ} \mathrm{C}$, ambiente fresco, alivianar cobertores. 
9.1.3. Posición semifowler con cabeza ladeada para prevenir as piración de vómitos.

\subsubsection{Aseo cavidad bucal según necesidad.}

9.1.5. Registro de cantidad y calidad del vómito.

\subsubsection{Evaluar hidratación del paciente.}

10. Incapacidad fisiológica y/o patológica para sat isfacer sus necesidades básicâs.

\subsection{Cuidados de enfermería}

\subsubsection{Confort del paciente.}

10.1.2. Administración de alimentación según necesidad.

10.1.3. Administración de hidratación según necesidad.

Registrar en forma completa y oportuna la atención y observaciones de enfermería brindadas al paciente, y evaluar en cada entrega de turno los cuidados de enfermería.

Finalmente pensamos que este paciente debe ser atendido por el profesional de enfermería, quien está preparado para satisfacer las necesidades y ayudar a resolver los problemas del niño con incapacidad para participar en actividades normales.

\section{SUMMARY}

22 clinical cases of Guillain-Barré syndrome were detected in the Infectious unit of the Pediatric area at the Regional Hospital, Antofagasta since 1972 to March 1979. Five of the patients died, two of them belonged to the Landry modality.

Since there is scarce bibliographic information about this syndrome here, and according to the previously described casuistry, it is a challenge for the nurse professional to give an adequate attention to many of the problems that affect this kind of patients and to a permanent risk of disease complications that in its worst degree may lead them to death. This fact motivates the professionals to present an attention plan for these children.

Considering that this syndrome is a acute polirradiculoneuritis with albumino-citological of LCR dissociation which is revealed as an acute infectious chart of progressive evolution, with motor and sensitive alterations, myalgia, cephalea, vasomotor alterations, temporary sphincteral alterations, irritability and drowsiness: this patient will be evaluated as an individual whose pathologic physical appearance is recoverable with a light psychicalteration due to the apprehension of the problems that affect him.

Complications in 22 Guillain-Barré clinical cases: Infectious Unity. Pediatric area Regional Hospital, Antofagasta 1972 - March 1979.
In these twenty-two clinical cases the most frequent problems the Nurse professional had to assist were the motor complications $(51,3 \%)$ pains $(19.1 \%)$ and the vasomotor alterations $(8.6 \%)$ therefore the nurse plan should be mainly aimed at the attention and constant control of these manifestations without neglecting the observation and attention of the other alterations presented by the patient.

We must emphasize that $22.7 \%$ of these patients presented respiratory alterations and $18.1 \%$ presented muscular atrophy. The nurse assistance that the patients received during the hospitalization was $68.1 \%$ unspecified and only $31.8 \%$ of them received a specified nurse assistance to their necessities, nevertheless the total universe, that is to say $45.4 \%$ of the nurse observations, was in relation to the problems of these patients.

According to the present data, it is concluded that the most suitable nurse assistance for these patients is summarized as folkows:

1. The nurse assistance, awaiting the confirmed medical diagnosis, is the isolation and the use of isolation techniques until the differential diagnosis of poliomyelitis is established, then blood and defecation samples will be taken by the nurse to perform a virologic study and to help the doctor in obtaining LCR sample.

2. To observe and evaluate the sensibility and motor function of the affected limbs.

3. Hemodynamic evaluation through the pulse control, arterial pressure and skin coloration.

4. Control of the respiratory dynamic by means of rhythm evaluation, frequency and respiratory movements.

5. Prevention of perverted positions and muscular atrophy.

6. Prevention of decubitus ulcers and pain relief.

7. To offer emotional support and provide a proper environment to their emotional needs.

8. Constant observation in the search of sphincteral alterations.

9. Observation and control of the patient's general state in search of other signs and/or symptoms such as fever, vomits, etc. Finally, these patients should be attended in specialized units by nurse professionals who should perform complete and opportune reports of nurse assistance and observations offered to the patient

\section{REFERENCIAS}

1 Farreras, P.; Rozman Ciril. Medicina Interna. Tomo II. $8^{\circ}$ Edición. Editorial Marín S.A. 1972, Cap. $6^{\circ}$, págs.: 137 a 138 .

2 Meneghello, J. Pediatria. Tomo II. Editorial Inter Médica. Bs. Aires, Argetina, 1972, págs. 495-496.

3 Kempe, H.; Silver, H.; O'Brien, D. Diagnóstico y tratamiento pediátrico. Editorial EJ Manual Moderno S.A. $1^{\star}$ Edición, págs. 498-499.

4 Harrison. Medicina Interna. Editorial La Prensa Mexicana. $5^{a}$ Edición en espan̄ol, 1973, págs. 19001901.

5 Brunner; Emerson; Ferguson. Enlermería Médico Quirúrgica. $2^{a}$ Edición. Editorial Interamericana, 1971. Cap. X, págs. 170-171, 176, 895, 179. 
Kozier, Dugas. Tratado de Enfermería práctica. 2 Edición. Editorial Interamericana, 1974. Cap. XX, págs. 225 a 229; Cap. XXIv, págs. 290 a 293.

7 Eberle, E., MD. Brink, J., MD. Early prediction of incomplete recovery in children with Guillain-Barré polyneuritis. The Journal of Pediatrics. March 1975. Volumen 86. N 3, págs. 356-357.

* Varela, N. Dr. El Sindrome de Guillain-Barré. Boletin del Hospital San Juan de Dios. Vol. 10, 1963.

- Informe Epidemiológico Semanal. El Sindrome de Guillain-Barré. Oficina Sanitaria Panamericana. Vol. 49. $N^{\circ} 1,5$ enero 1977.

10 Debré R., Thieffory S. Remarques sur le Syndrome de Guillain-Barré chez l'enfant. Hospital de niños, Paris, Francia.

11 Peterman A., Daly D. Guillain-Barré en niños (Clinica Mayo). Apartado de la $11^{*}$ reunión anual de la academia americana de neurología, Los Angeles, California, 1959.

1.2 Aning Charles, Sabin Albert. Art. del Laboratorio de Neuropatología del Hospital General de Cincinnati.

13 Grase C., M.D. Spigland J, M.D. División de Inmunología Pediátrica, Universidad de California, San Francisco. División de Virologia y Depto. de Patología y Pediatría. Centro Médico y Hospital de Montefiore y Escuela de Medicina Albert Einstein, New York. 